\title{
Factors contributing to defaulting scheduled therapy sessions by caregivers of children with congenital disabilities
}

\section{A Nota $^{1}$, T M Chikwanha ${ }^{2}$, J January ${ }^{3 *}$, N Dangarembizi}

1. Chitungwiza Central Hospital, Zimbabwe

2. Department of Rehabilitation, College of Health Sciences, University of Zimbabwe

3. Department of Community Medicine, College of Health Sciences, University of Zimbabwe

Correspondence to: Mr. James January

Email: jamesj@medsch.uz.ac.zw

\[ \text { Abstract } \]
Background
Defaulting scheduled rehabilitation therapy may result in increased
adverse outcomes such as permanent disability and increased healthcare
costs. Concomitantly, there is evidence to suggest that early and continued
rehabilitation of children with congenital disabilities can improve
outcomes significantly. This study was conducted to determine factors
contributing to caregivers' defaulting scheduled rehabilitation therapy
sessions.

Methods

A descriptive cross sectional study was carried out at Chitungwiza Central Hospital, a tertiary facility offering in and outpatient rehabilitation services in Zimbabwe. Caregivers of children who had congenital disabilities $(\mathrm{N}=40)$ and who had a history of defaulting treatment but were available during the data collection period responded to an interviewer administered questionnaire. Data were analysed for means and frequencies using STATA 13.

\section{Results}

Factors that contributed to caregivers defaulting scheduled therapy included economic constraints (52\%), child related factors (43\%), caregiver related factors $(42 \%)$, service centred factors $(30 \%)$ and psychosocial factors $(58 \%)$. Majority of the caregivers $(98 \%)$ were motivated to attend therapy by observable improvements in their children. Other motivators were incentives given in the rehabilitation department (45\%), availability of rehabilitation personnel to provide the required services $(48 \%)$ and psychosocial support from fellow caregivers, families and the rehabilitation staff $(68 \%)$. Although all the caregivers could not distinguish occupational therapy from physiotherapy services they all reported that therapy was important.

\section{Conclusions}

A combination of psychosocial, economic, child centred and service centred factors contributed to caregivers defaulting scheduled therapy. Interventions that may potentially improve caregiver attendance to scheduled therapy include community outreach services, efficient rehabilitation service provision at the hospitals, and facilitation of income generating programmes for caregivers.

\section{Introduction}

Disabilities in children manifest in different debilitating symptoms which may vary from mild to severe limitations in terms of activity performance, participation restrictions and impairments ${ }^{1}$. Children with disabilities depend entirely on their primary caregiver when accessing rehabilitation services. It is generally agreed that caring for children with complex disabilities poses excess psychological ${ }^{2,3}$, physical, and economic strain on the caregiver ${ }^{4}$.

Children with disabilities need early and consistent rehabilitation services so that they can be able to reach their maximum capabilities in life ${ }^{5}$. Rehabilitation of children with disabilities should thus consider family centred service as a way to increase participation of children with disabilities ${ }^{6}$. This can only be achieved if the child attends scheduled rehabilitation therapy sessions in addition to also meeting the needs and expectations of primary caregivers ${ }^{7}$; a feat that is equally challenging in resource constrained settings such as Zimbabwe. Rehabilitation of children with disabilities is a process that requires time to produce results, and better treatment outcomes are achieved when early identification and interventions are holistically instituted ${ }^{8}$. It is therefore crucial to incorporate primary caregivers in the overall management of the patient. In Zimbabwe, rehabilitation services are found at district, provincial and tertiary health centres. Provision of rehabilitation services is in line with the primary health care approach ${ }^{9}$. At the district health centres, rehabilitation services are provided by rehabilitation technicians, who are middle-level rehabilitation professionals trained to provide basic occupational therapy, physiotherapy and speech therapy services in the absence of occupational therapists, physiotherapists and speech therapists respectively. The provincial health centres have both rehabilitation technicians and therapists. The tertiary institutions are mainly referral centres although they can also receive cases which are normally seen at district and provincial levels of care. When patients visit the rehabilitation department for therapy they are all entered into the rehabilitation register and based on the therapists' treatment plans those needing subsequent visits are given follow up appointments. These patients are then entered into the rehabilitations department's appointment registers, which makes it easier to track those who will be missing scheduled therapy sessions.

Although rehabilitation services at all tertiary institutions in Zimbabwe are offered free of charge to children under five years, statistics from the rehabilitation departments at these tertiary institutions for the period 2011 2013 indicated that some of the caregivers defaulted scheduled individual therapy sessions, group therapy and scheduled caregiver workshops ${ }^{10}$. Literature indicates that psychosocial, economic, child centred and service centred factors are major contributors to caregiver defaulting therapy ${ }^{11}$. However, the factors contributing to caregivers defaulting scheduled therapy in the Zimbabwean setting were not fully understood. Therefore a study was conducted to determine factors contributing to caregivers of children with congenital disabilities defaulting scheduled therapy sessions.

\section{Methods}

Ethical approval for the study was sought from the University of Zimbabwe, College of Health Sciences Ethics and Research Committee (JREC 312/13), the Medical Research Council of Zimbabwe (MRCZ/B/602) and from Chitungwiza Central Hospital where the research was conducted. All caregivers gave written consent before participating in the study. A descriptive cross sectional study was carried out at Chitungwiza Central Hospital, a tertiary facility offering in-patient and outpatient rehabilitation services. The hospital has one rehabilitation department offering inpatient and out-patient occupational therapy, physiotherapy and speech therapy services. The department currently has six physiotherapists, five occupational therapist and seven rehabilitation technicians. Approximately 260 patients receive rehabilitation services at the hospital every month, with 110 of these being children with congenital deformities ${ }^{10}$. In the past two years approximately sixty children with neurological conditions and thirty children with orthopaedic conditions were being seen every month in the rehabilitation department. Weekly clinics are conducted every Monday for children with orthopaedic conditions and on Wednesdays for children with neurologic conditions. However the frequency of review dates is determined by the rehabilitation practitioners as per individual patient needs

The study sample comprised of male and female adult caregivers who had a child aged five years and below with a congenital disability. We included caregivers aged above 18 years and were resident in Chitungwiza. For the purposes of this study defaulting was defined as missing at least one scheduled therapy appointment. Caregivers who were not the primary caregiver and were unable to communicate in Shona or English were excluded from the study. Caregivers of children with disabilities who had a history of defaulting were identified through the rehabilitation registers. They were then approached as they were coming for their reviews. Those caregivers who were available at the time of data collection and had a history of defaulting treatment were invited to take part in the study. An interviewer administered questionnaire was used to collect data from the caregivers. The questionnaire was specifically designed by the researchers for the Zimbabwean setting using information available in the reviewed literature. The questionnaire collected information on the demographic 
characteristics of participants; rehabilitation services accessed by the caregivers at Chitungwiza central hospital, perceived importance of the rehabilitation services, factors that contributed to defaulting therapy and also the caregivers suggestions on the strategies that could be put in place to prevent defaulting as well as other rehabilitation services that were needed by the caregivers. The questionnaire was designed in English and then translated into the local Shona language. A pre-test was conducted before the actual study at Harare Central Hospital children`s rehabilitation Unit (CRU) with the caregivers of patients aged below five years and had previously defaulted treatment. From the results of the pre-test, it was noted that the caregivers had no problems understanding the questions and the results also showed that the instrument was capturing the information that was in line with set objectives. Therefore no changes were made to the original questionnaire. Data were collected over a period of 2 months. Quantitative data were analysed for means and frequencies using STATA 13 statistical package (STATA Corporation, College Station, Texas, USA)

\section{Results}

All the forty caregivers found eligible were invited and agreed to take part in the study giving a response rate of $100 \%$. Thirty-nine $(98 \%)$ of the caregivers were females and only one was male. Majority $(75 \%)$ were aged between 20 and 39 years. Thirty-one (78\%) of the caregivers were biological parents while the rest were grandparents, aunts or cousins. Eight $(20 \%)$ of the caregivers were formally employed, with the other $80 \%$ being either informally employed or unemployed. Only two $(4 \%)$ of the caregivers reported that they had other children with disabilities. The mean age of the children cared for by caregivers who participated in this study was 1.5 years $(\mathrm{SD} \pm 1)$. Twenty five $(62 \%)$ of the children were males while $38 \%$ percent were females. Twenty-four $(60 \%)$ of the children had neurological conditions and sixteen $(40 \%)$ had orthopaedic conditions. Neurological conditions included cerebral palsy, hydrocephalus and spinal bifida. Orthopaedic conditions included clubfoot, external hip rotations and arthrogryposis multiplex.

\section{Rehabilitation services accessed by the caregivers}

All the caregivers reported that they had accessed either physiotherapy or occupational therapy services. However all participants had challenges in distinguishing between occupational therapy, speech therapy and physiotherapy services and referred to them as exercises. The specific services accessed included provision of required aids and appliances, individual therapeutic treatment sessions, family counselling, group sessions and caregiver workshops. Six $(15 \%)$ of the caregivers had also accessed speech therapy services. All the participants perceived rehabilitation services as important in the management of children with disabilities. Reasons mentioned for this perceived importance were the child`s observable progress and reduction of caregiving burden.

\section{Motivators to attending scheduled therapy sessions as reported by caregivers}

Observable improvements in the children's condition were reported by thirty nine $(98 \%)$ of the caregivers to be a motivator for accessing rehabilitation services. Improvements noted in the children included attainment of developmental milestones for those with neurological conditions and corrections of deformities for those with orthopaedic conditions. Incentives given in the rehabilitation department such as aids and appliances, toys, food, clothes and money for transport during workshops were also reported as motivators by eighteen $(45 \%)$ of the caregivers. Availability of rehabilitation personnel to provide the required services each time they visited the rehabilitation department motivated another nineteen $(48 \%)$ of the caregivers. Twenty seven $(68 \%)$ of the caregivers were motivated by psychosocial support from fellow caregivers, families and the rehabilitation staff. Caregivers exchanged ideas on issues pertaining to the upkeep of their children when they met in the rehabilitation department either during therapy sessions or during caregiver educational workshops. Emotional support received from fellow caregivers was reported as a motivator by nineteen $(48 \%)$ of the caregivers.

\section{Factors contributing to caregivers defaulting treatment sessions}

Individual treatment sessions had the greatest $(52 \%)$ number of defaulters compared to workshops and group sessions. There were more $(60 \%)$ caregivers of children with neurologic conditions defaulting treatment compared to those of children with orthopaedic conditions $(40 \%)$. Economic challenges such as lack of bus fare to get to the hospital were reported by twenty one $(52 \%)$ of the caregivers. The nature of the disability made it difficult for some of the caregivers $(n=17)$ to transport their children to the hospital. Another seventeen (42\%) reported that they forgot appointment dates. Those caregivers who were formally employed $(20 \%)$ reported that it was difficult to ask for time off work each time they had to attend therapy with their child. Another factor cited by $30 \%$ of the caregivers related to the long waiting times in the rehabilitation department during the children`s clinics. Social stigma was reported by twenty-three (58\%) of the caregivers to be another factor that contributed to them missing scheduled appointments.

\section{Strategies for improving caregiver attendance to scheduled therapy sessions as recommended by the caregivers}

The strategy that was advocated for by most $(80 \%)$ of the caregivers was increasing individual therapy sessions with more time per session. They also felt that individual therapy sessions would allow the caregivers enough time to consult with the therapists on areas where they needed more help. Thirty $(75 \%)$ of caregivers reported that they needed more aids and appliances to use in the department and at home with their children. Twenty three $(58 \%)$ of the caregivers also advocated for the establishment of a specialised children's rehabilitation unit at Chitungwiza Central Hospital. Fourteen $(35 \%)$ of the caregivers reported that they needed resources to set up income generating projects as the money generated would then assist them with transport costs, purchase of aids and appliances and general upkeep of these children.

\section{Discussion}

Although this cross-sectional study was confined to only one of the five tertiary institutions offering rehabilitation services in Zimbabwe the results obtained demonstrate the profound importance attached to rehabilitation of children with disabilities. Despite all the caregivers having had missed at least one scheduled therapy session, they all concurred to the importance of rehabilitation for their children. Various factors which included economic, psychosocial, environmental, caregiver-centred, service provider centred and child centred factors were highlighted as contributing to caregivers defaulting scheduled therapy sessions. The data gathered in this study seem to suggest that these factors are entangled in each other and should thus not be considered in isolation. In resource constrained settings, there is usually an interplay of socio-economic difficulties, inadequate resources, dissatisfied services providers and social stigma towards physical disabilities and socio-political and cultural histories all of which make it arduous for caregivers to adhere to scheduled therapy sessions ${ }^{12}$. Caregivers in our study could not specifically pin point whether they had accessed physiotherapy, occupational therapy or speech therapy services. The rehabilitation services at Chitungwiza are offered under one roof therefore the caregivers may have found it difficult to differentiate between the different types of services. However providing all the rehabilitation services under one roof fosters a holistic approach to treatment .This is supported by Siebes and colleagues ${ }^{8}$ who reported that a holistic approach is important in the management of children with disabilities. The importance attached to having holistic and consistent rehabilitation for children with disabilities that was noted by the caregivers in this study has also been previously echoed in other populations ${ }^{13}$. It is noteworthy to mention that caregivers defaulted individual therapy more as compared to group therapy and workshops. Attending rehabilitation sessions enabled caregivers to discuss and share experiences with each other and with rehabilitation staff. Based on the researchers' experiences working with caregivers, such an opportunity could have motivated them to continue accessing rehabilitation services. Furthermore, such interactions between caregivers and rehabilitation personnel foster better partnerships between the two ${ }^{14}$ which may ultimately translate into enhanced treatment outcomes for the children. In view of this finding, it is however ironic that the majority of caregivers in this study recommended that more time be increased for individual sessions. A plausible explication for this may be that the caregivers felt that the individual sessions were too short to have significant benefits and therefore they opted for group therapy sessions. Caregivers may also have favoured more group sessions and workshops as these provide a platform for them to share with other caregivers and offers an opportunity to "measure" their child's progress against others. Future studies could potentially look into why caregivers tended to prefer group therapy as compared to individual therapy. There is therefore need for continued health education to the caregivers on the benefits of attending both individual and group sessions. In addition, it is consequently imperative to integrate caregiver support groups and improve caregiverhealth personnel interactions in the overall rehabilitation of children with disabilities.

The provision of incentives by the rehabilitation department, in form of aids and appliances, assistive devices, toys, food, transport fares and 
clothes was also instrumental in motivating caregivers as revealed in this study. Reported benefits for such provision of aids and appliances included reduction of the caregiver burden as the children become more functionally independent. This is supported by Ripat and Woodgate (2012) who mentioned that assistive technologies contribute to participation of people with disabilities in activities of daily living ${ }^{15}$. With regards to defaulting therapy sessions, the caregivers reported that they encounter economic and service centred factors and resulted in them defaulting therapy. Caregivers of children with special needs experience financial difficulties in caring for their children and this in turn adversely impacts their attendance to scheduled therapy sessions ${ }^{4}$. This was further reiterated by O'Neil and her colleagues who posited that family financial difficulties were related to an increased time to provide healthcare for children with special needs ${ }^{16}$. In Zimbabwe, the hyperinflationary environment experienced in 2007-2008 eroded most people's savings and health insurances ${ }^{17}$. Despite the government of Zimbabwe offering free health services for children below 5 years, children with disabilities have other needs such as special food, assistive devices and transport fees to the hospital. Therefore income generating projects should be initiated for the caregivers to be self-sufficient ${ }^{9}$. About half of the caregivers reported that they had other children to look after at home and only a few had other children with disabilities. Performing duties related to caring for a child with disabilities is time consuming ${ }^{16}$ and long waiting times could have been taxing for the caregivers. Long waiting queues may have contributed to caregivers defaulting therapy. This could be minimized if the rehabilitation department could book patients which they could handle at specified times and also attending to these children as soon as possible so that the caregivers can go back early. Child related factors were also reported by the caregivers as contributing to defaulting scheduled therapy sessions. These included burden of transporting the child to and from the rehabilitation department for therapy. Most caregivers of children with disabilities may have physical difficulties when assisting the children with mobility and self-care activities ${ }^{18}$. Custom made wheelchairs are beyond the reach of many Zimbabweans, and caregivers rely on donated wheelchairs which are sometimes ill-fitting and result in development of complications. In addition, bad terrain makes it difficult to use the wheelchairs to transport those children who were on wheelchairs to the hospital ${ }^{11}$. Most of the strategies recommended by the caregivers to improve attendance for scheduled therapy can best be addressed by establishing a unit that is solely for children. Currently the rehabilitation department at the hospital provides services to both adults and children. In other central hospitals in Zimbabwe, the children`s rehabilitation units operate as an extension of the main rehabilitation department with services that are more child oriented $^{19}$. The services offered include physiotherapy, occupational therapy, workshops and group counselling, medical clinics and caregiver training ${ }^{19}$. The therapists working in a sorely child-oriented rehabilitation unit will have more time for individual sessions and for following up the children in their respective communities.

\section{Recommendations for future studies}

This study provides an important baseline for conducting bigger studies whose findings will be more generalizable. The samples for the bigger studies could include caregivers who had not defaulted treatment to get a comparison and wider view of the factors that affect adherence to scheduled therapy sessions. In order to gain a deeper understanding of the way caregivers perceive the challenges that they face when accessing services, qualitative methodologies may be employed as they have the advantage of bringing to the core inner perceptions and lived experiences.

\section{Conclusion}

Despite the limitations to the study, the results from this study lend weight to the notion that a variety of factors contribute to defaulting therapy among caregivers of children with congenital disabilities. This paper has gone some way in exploring defaulting for scheduled therapy and caregiver perceptions of rehabilitation services for children with disabilities in Zimbabwe. It has highlighted service related as well as caregiver related challenges which calls for greater need to provide comprehensive and accessible rehabilitation services for this vulnerable group. Caregivers had physical, economic and psychosocial challenges that made it difficult to access rehabilitation services on scheduled therapy dates, which resulted in them defaulting therapy. Interventions that need to be strengthened include community outreach services, efficient rehabilitation service provision at the hospitals, and facilitation of income generating programmes for caregivers.

\section{Acknowledgements}

Chitungwiza Hospital clinical director and rehabilitation staff.
All the caregivers.

University of Zimbabwe College of Health Sciences Department of Rehabilitation.

\section{References}

1. Allet L, Bürge E, Monnin D. ICF: Clinical relevance for physiotherapy? A critical review. Adv Physiother. 2008 Jan;10(3):12737.

2. Koehler AD, Fagnano M, Montes G, Halterman JS. Elevated Burden for Caregivers of Children with Persistent Asthma and a Developmental Disability. Matern Child Health J. 2014 Mar 12;1-9.

3. Gallagher S, Hannigan A. Depression and chronic health conditions in parents of children with and without developmental disabilities: The growing up in Ireland cohort study. Res Dev Disabil. 2014 Feb;35(2):448-54

4. Rofail D, Maguire L, Kissner M, Colligs A, Abetz-Webb L. A Review of the Social, Psychological, and Economic Burdens Experienced by People with Spina Bifida and Their Caregivers. Neurol Ther. 2013 Dec $1 ; 2(1-2): 1-12$

5. Guralnick MJ, Bricker D. The effectiveness of early intervention for children with cognitive and general developmental delays. [Internet]. San Diego, CA: Academic Press; 1987 [cited 2014 Aug 1]. Available from: http://psycnet.apa.org/psycinfo/1987-97527-004

6. Piškur B, Beurskens AJHM, Jongmans MJ, Ketelaar M, Smeets RJEM. What do parents need to enhance participation of their schoolaged child with a physical disability? A cross-sectional study in the Netherlands. Child Care Health Dev. 2014 Jun 1;n/a - n/a.

7. Piškur B, Beurskens AJ, Jongmans MJ, Ketelaar M, Norton M, Frings CA, et al. Parents' actions, challenges, and needs while enabling participation of children with a physical disability: a scoping review. BMC Pediatr. 2012 Nov 8;12(1):177.

8. Siebes RC, Wijnroks L, Ketelaar M, Van Schie PEM, Gorter JW, Vermeer A. Parent participation in paediatric rehabilitation treatment centres in the Netherlands: a parents' viewpoint. Child Care Health Dev. 2007 Mar 1;33(2):196-205.

9. Ministry of Health \& Child Welfare. The National Health Strategy for Zimbabwe (2009-2013). Government of Zimbabwe, Harare; 2009.

10. Ministry of Health \& Child Care. Rehabilitation Monthly Departmental Returns 2011-2013. 2013

11. Resch JA, Mireles G, Benz MR, Grenwelge C, Peterson R, Zhang D. Giving parents a voice: A qualitative study of the challenges experienced by parents of children with disabilities. Rehabil Psychol. 2010 May;55(2):139-50.

12. Mpofu E, Harley DA. Disability and Rehabilitation in Zimbabwe: Lessons and Implications for Rehabilitation Practice in the US (Disability and Rehabilitation in Zimbabwe). J Rehabil. 2002;68(4):26.

13. Maloni PK, Despres ER, Habbous J, Primmer AR, Slatten JB, Gibson BE, et al. Perceptions of disability among mothers of children with disability in Bangladesh: Implications for rehabilitation service delivery. Disabil Rehabil. 2010 Jan 1;32(10):845-54.

14. Järvikoski A, Härkäpää K, Martin M, Vasari P, Autti-Rämö I. Service characteristics as predictors of parents' perceptions of child rehabilitation outcomes. J Child Health Care. 2013 Oct 3;1367493513503579.

15. Ripat JD, Woodgate RL. The role of assistive technology in selfperceived participation: Int J Rehabil Res. 2012 Jun;35(2):170-7.

16. O'Neil ME, Costigan TE, Gracely EJ, Wells N. Parents' Perspectives on Access to Rehabilitation Services for Their Children with Special Healthcare Needs: Pediatr Phys Ther. 2009;21(3):254-60.

17. Hanke SH, Kwok AKF. On the Measurement of Zimbabwe's Hyperinflation. Cato J. 2009;29:353. 
18. Andrews M, Bolt DM, Braun M, Benedict RE. Measuring Exertion During Caregiving of Children and Young Adults with Cerebral Palsy Who Require Assistance for Mobility and Self-Care. Phys Occup Ther Pediatr. 2013 Jan 29;33(3):300-12.
19. Jelsma J, Cortes-Meldrum D, Moyo A, Powell G. The Children's Rehabilitation Unit, Harare, Zimbabwe: An Integrated Model of Rehabilitation. Pediatr Phys Ther. 1995;7(3):140-2. 